\title{
Adaptive Speed Control for Permanent Magnet Synchronous Motor with NLMS Parameters Estimation
}

\author{
Jiang Xuecheng ${ }^{1}$ and He Dongwei ${ }^{2}$ \\ ${ }^{1}$ Dept. of Electronic, Minjiang University, Fuzhou, P. R. China \\ ${ }^{2}$ Dept. of Electronic Information, Fujian University of Technology, Fuzhou, \\ P. R. China \\ ${ }^{1}$ jxc603@aliyun.com, ${ }^{2} y z a k \_j u e l @ q q . c o m$
}

\begin{abstract}
Load torque disturbance and the variations of parameters are contained in speed discrete time control system for permanent magnet synchronous motor (PMSM). In order to improve the robustness of PMSM control system, an adaptive control scheme based on normalized least mean square (NLMS) algorithm for parameters estimation is proposed in this paper. The sufficient condition satisfied with model adaptive compensation is deducted in detail. Additionally, get the compensation functions by the value of estimating parameter and the control scheme can drive the system controlled output to track the command like the nominal plant. Finally, the stability and rapidity of this control scheme are fully verified by the experiment of PMSM speed control system.
\end{abstract}

Keywords: permanent magnet synchronous motor, normalized least mean square algorithm, parameters estimation, adaptive control

\section{Introduction}

In many industrial drives, advanced digital control strategies for the control of fieldoriented PMSM drives with a conventional speed controller, proportional-integral (PI) controller, have gained the widest acceptance in high-performance ac servo systems [1]. Due to the existence of load torque disturbance and variable parameters for PMSM control system, many papers have been published on the on-line and off-line motor parameters estimation algorithms [2-5]. Adaptive control based on parameters estimation can overcome well load torque disturbance and variable parameters [6-8]. Load torque is looked as state variables and estimated by the reduction observer of the augmented normal system, then the estimated load torque feed-forward compensates PI controller for PMSM speed system [9]. The disturbance rejection can also be achieved based on a multiobjective observer in which observation error is purposely retained and utilized in load torque disturbance compensation [10]. However, in both methods other variable parameters are regarded as a constant. So in [11] the neural network identification estimates variable parameters and another neural network estimates load torque. In [12] the model-following error is used to adaptively update the gains of the PI controller and a disturbance observer based on the time delay control approach is used for torque feed forward control.

PMSM speed discrete time control system is a linear system and may be described by the controlled autoregressive moving average model (CARAM). The system parameters and load torque can be estimated from available input-output data. This paper presents an adaptive control scheme based on NLMS algorithm that estimates the parameters and load torque of PMSM at the same time. Get the compensation functions by the value of estimating parameters and the control scheme can drive the system controlled output to track the command like the nominal plant. And the sufficient condition satisfied with model adaptive compensation is also deducted and result of the experimental shows that 
this control scheme can enhance the robustness of the controller and make system rapidly track the command speed signal.

\section{PMSM Online Parameters Estimation}

The discrete time SISO control linear system, only considering the perturbations of the state variable data, the controlled object parameters estimation will be simple. The implication is illustrated by the solution of a CARAM equation

$$
\begin{aligned}
& y(k)=x^{\mathrm{T}}(k-d) \theta+\sigma(k) \\
& x^{\mathrm{T}}(k-d)=\left[y(k-1), y(k-2) \ldots y\left(k-n_{a}\right), u(k-1), u(k-2) \ldots u\left(k-n_{b}\right)\right] \\
& \theta=\left[\alpha_{1}, \alpha_{2} \ldots \alpha_{n_{a}}, \beta_{1}, \beta_{2} \ldots \beta_{n_{b}}\right]^{\mathrm{T}}, n_{a}+n_{b}=n
\end{aligned}
$$

Where $x(k) \in R^{n}$ is the state variable matrix and $y(k) \in R$ is the output vector. $\sigma(k)$ is white noises known means and variance. $\theta \in R^{n}$ is the controlled object parameters vector, $k$ is time sequence and $d$ is system delay time. The error of parameters estimation

$$
\hat{e}(k)=y(k)-x^{\mathrm{T}}(k-d) \hat{\theta}(k)
$$

Thus, the parameters vector estimation problem can be obtained by solving the optimization problem

$$
\min \|\hat{\theta}(k+1)-\hat{\theta}(k)\|^{2}, y(k)-x^{\mathrm{T}}(k) \hat{\theta}(k+1)=0
$$

Considering Multi constrained Lagrange operator method, the following information criterion defines as

$$
J(k)=\|\hat{\theta}(k+1)-\hat{\theta}(k)\|^{2}+\sum_{i=0}^{P-1} \lambda_{i}\left[y(k-i)-x^{\mathrm{T}}(k-i-d) \hat{\theta}(k+1)\right]
$$

Where $\lambda$ is the Lagrange multiplier. The $\hat{\theta}(k+1)$ derivative of the function (4) is

$$
\frac{\partial J(k)}{\partial \hat{\theta}(k+1)}=2(\hat{\theta}(k+1)-\hat{\theta}(k))-x(k) \lambda
$$

According to [13], an iterative NLMS algorithm for parameters estimation is

$$
\hat{\theta}(k+1)=\hat{\theta}(k)+\mu x(k)\left[x^{\mathrm{T}}(k) x(k)\right]^{-1} e(k)
$$

Where $\mu$ is a regularization factor that keep sufficient condition for convergence and the range is $0 \leq \mu \leq 1$.

In rotating coordinate based field-oriented axes, the model of PMSM can be expressed as follows

$$
\left\{\begin{array}{l}
u_{\mathrm{d}}=R_{\mathrm{s}} i_{\mathrm{d}}+p L i_{\mathrm{d}}-P_{n} \omega_{\Omega} L i_{\mathrm{q}} \\
u_{\mathrm{q}}=R_{\mathrm{s}} i_{\mathrm{q}}+p L i_{\mathrm{q}}+P_{n} \omega_{\Omega}\left(L i_{\mathrm{d}}+\psi_{\mathrm{f}}\right) \\
T_{\mathrm{e}}=p J \omega_{\Omega}+B \omega_{\Omega}+T_{\mathrm{L}}
\end{array}\right.
$$

Where $u_{\mathrm{d}}$ and $u_{\mathrm{q}}$ are the $\mathrm{d}-\mathrm{q}$ axis stator voltages, $i_{\mathrm{d}}$ and $i_{\mathrm{q}}$ are the $\mathrm{d}-\mathrm{q}$ axis stator currents, $\mathrm{L}$ and $R_{\mathrm{s}}$ are the d-q axis stator inductances and the stator resistance, $\omega_{\Omega}, T_{\mathrm{e}}$, $P_{\mathrm{n}}$ and $P$ are the mechanical angular velocity, the motor torque respectively, motor pole pairs and the differential operator respectively. $\psi_{\mathrm{f}}, J, B$ and $T_{\mathrm{L}}$ are the flux linkage of the rotor magnets, the inertia momentum, the damping factor and the load torque 
respectively and they are the estimated parameters of PMSM speed loop in this paper. The discrete equation (7) for PMSM speed loop can be expressed as

$$
\omega_{\Omega}(k)=\alpha \omega_{\Omega}(k-1)+\beta i_{\mathrm{q}}(k)-\gamma T_{\mathrm{L}}(k-1)
$$

Where $\alpha=e^{\left(-B T_{s} / J\right)}, \beta=P_{\mathrm{n}} \psi_{\mathrm{f}}(1-\alpha) / B, \gamma=\beta / P_{\mathrm{n}} \psi_{\mathrm{f}}, T_{s}$ is discrete sampling period. The load torque is time variant and is difficult to measure directly. It is better that $\alpha, \beta, \gamma$ and $T_{\mathrm{L}}$ can finish online real-time estimating at the same time. So the online parameters estimation structure of the PMSM speed loop is shown on Figure 1.

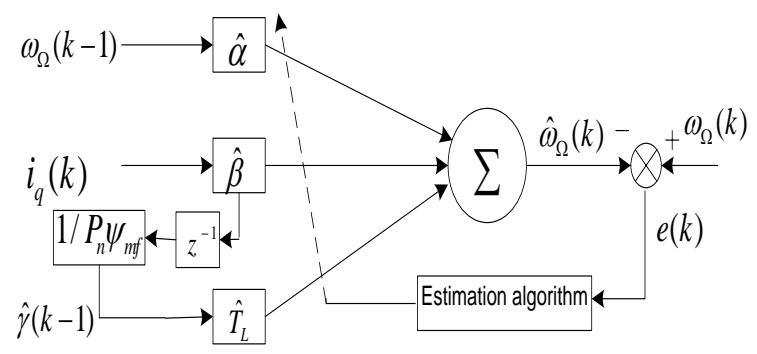

\section{Figure 1. Structure of Online Parameters Estimation}

In the PMSM speed control system, get the speed $\omega_{\Omega}(k)$ by differential calculation using the photoelectric position sensor, and then $i_{q}(k)$ by the current sensor. The state variable matrix and the output vector of parameters estimation algorithm both have the perturbations. Hypothesize

$$
\left\{\begin{array}{l}
\hat{\theta}(k)=\left[\begin{array}{lll}
\hat{\alpha}(k) & \hat{\beta}(k) & \hat{T}_{L}(k)
\end{array}\right] \\
x(k)=\left[\begin{array}{lll}
\omega_{\Omega}(k-1) & i_{q}(k) & -\hat{\gamma}(k-1)
\end{array}\right] \\
\omega_{\Omega}(k)=x(k) \boldsymbol{\theta}^{\mathrm{T}}(k)+v(k)
\end{array}\right.
$$

In conclusion, $\hat{\gamma}=\hat{\beta} / P_{\mathrm{n}} \psi_{\mathrm{mf}}$ is defined as the state variable and $\hat{T}_{\mathrm{L}}$ as the estimated parameters. Substituting (9) into (6), we can get the estimation of PMSM parameters.

\section{PMSM Speed Adaptive Control}

The proposed control scheme consists of a conventional PI controller, the compensation function $\mathrm{G}_{1,2}$ and online parameters estimation algorithm. Get the compensation functions by the estimating value of the PMSM parameters. They are used to compensate the PI controller designed on the basis of the nominal plant.

Theorem: For the adaptive control for the PMSM speed loop as shown in Figure 2, provided that satisfying the following condition

$$
\hat{\gamma}=\hat{\beta} / P_{\mathrm{n}} \psi_{\mathrm{mf}}, G_{1}=\beta_{\mathrm{m}} / \hat{\beta}, G_{2}=\left(\alpha_{\mathrm{m}}-\hat{a}\right) / \hat{\beta}
$$




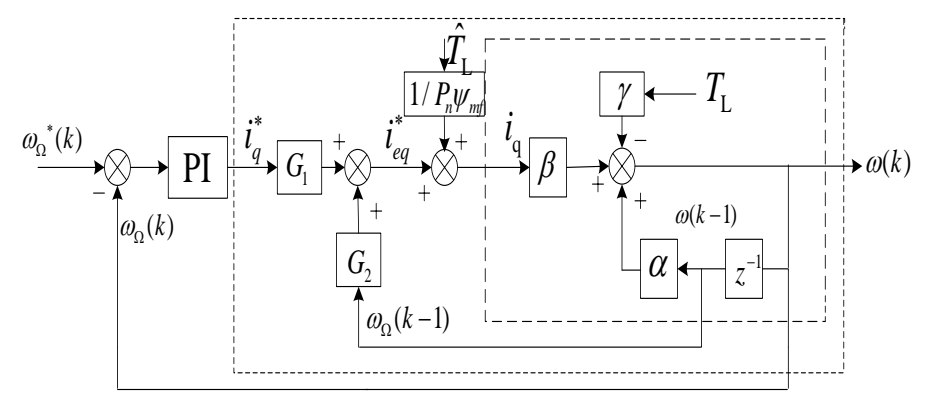

Figure 2. Structure of Adaptive Control

Then the PMSM speed control system can drive the system controlled output to track the command like the nominal plant

$$
\omega_{\Omega}(k)=\alpha_{\mathrm{m}} \omega_{\Omega}(k-1)+\beta_{\mathrm{m}} i_{\mathrm{q}}^{*}(k)
$$

Proof: By calculation of the online parameters estimation algorithm, the estimated parameters must be satisfied with

$$
\omega_{\Omega}(k) \approx \hat{\alpha} \omega_{\Omega}(k-1)+\hat{\beta} i_{\mathrm{q}}(k)-\hat{\gamma} \hat{T}_{\mathrm{L}}(k-1)
$$

From Figure 2, the $\mathrm{q}$ axis stator current is expressed as

$$
i_{\mathrm{q}}(k)=i_{\mathrm{q}}^{*}(k) G_{1}+\omega_{\Omega}(k-1) G_{2}+\hat{T}_{\mathrm{L}}(k-1) / P_{\mathrm{n}} \psi_{\mathrm{mf}}
$$

Substituting (13) into (12), the q-axis current is

$$
\omega_{\Omega}(k) \approx\left(\hat{\alpha}+\hat{\beta} G_{2}\right) \omega_{\Omega}(k-1)+\hat{\beta} G_{1} i_{\mathrm{q}}^{*}(k)+\hat{\beta} \hat{T}_{\mathrm{L}}(k-1) / P_{\mathrm{n}} \psi_{\mathrm{mf}}-\hat{\gamma} \hat{T}_{\mathrm{L}}(k-1)
$$

According to (10), noting that

$$
\omega_{\Omega}(k) \approx\left(\hat{\alpha}+\hat{\beta} G_{2}\right) \omega_{\Omega}(k-1)+\hat{\beta} G_{1} i_{\mathrm{q}}^{*}(k)=\alpha_{\mathrm{m}} \omega_{\Omega}(k-1)+\beta_{\mathrm{m}} i_{\mathrm{q}}^{*}(k)
$$

This completes the proof of Theorem.

Then, with the help of on-line adaptive compensation, the closed loop system is equivalent to nominal model without load torque disturbance and parameters variable, provided that the compensation functions and the parameters estimation algorithm satisfying equation (10) and (12). The estimated value of the parameters need not converge to the real value of the system parameters.

The specific steps of the proposed adaptive control can be described as follows

1)Initialize the value of $\hat{\alpha}, \hat{\beta}, \hat{\gamma}$ to the nominal value $\hat{\alpha}_{\mathrm{m}}, \hat{\beta}_{\mathrm{m}}, \hat{\gamma}_{\mathrm{m}}$ and the initial value of $\hat{T}_{\mathrm{L}}$ to zero

2)Design PI controller for the nominal plant

3)Processing in sampling internal, calculate $i_{\mathrm{q}}^{*}(k), \hat{\alpha}_{q}(k), \hat{\beta}_{q}(k), \hat{\gamma}_{q}(k)$ from (6) $\rightarrow$ calculate $G_{1} 、 G_{2}$ from (10) $\rightarrow$ calculate $i_{\mathrm{q}}(k)$ from (13)

4)Repeat the calculation of 3 )

\section{Experiment Results and Its Analysis}

PMSM with rated values are shown in Table 1. The maximum output current of $q$ axis stator is defined as $2.5 \mathrm{~A}$. In the proposed control scheme, $\mu$ is selected as 0.8 and the nominal value of $\alpha$ and $\beta$ are selected as 0.9998 and 1.3662 respectively. 
Table 1. PMSM Rated Parameters

\begin{tabular}{|l|l|l|l|}
\hline Rated power(W) & 200 & Rated speed(rpm) & 3000 \\
\hline Stator resistance $(\Omega)$ & 12.4 & Stator inductance $(\mathrm{mH})$ & 31.9 \\
\hline Moment of inertia(kg.cm2) & 0.167 & $\begin{array}{l}\text { The flux linkage of rotor } \\
\text { magnets }(\mathrm{wb})\end{array}$ & 0.0825 \\
\hline operating frequency of IPM $(\mathrm{kHz})$ & 5 & position optical encode(ppr) & 2500 \\
\hline
\end{tabular}

The overall experiment systems for PMSM speed control is shown in Figure 3. The systems created a time-varying load torque by adopting the magnetic powder brake in the experimental systems. It can help to demonstrate the effectiveness of the proposed control. The PI controller and the adaptive speed controller with field-oriented control are implemented using TMS320F28335. The system collects real-time experiment data of speed and parameters through TCP/IP communication, thereby verifying the merits of the proposed control scheme.

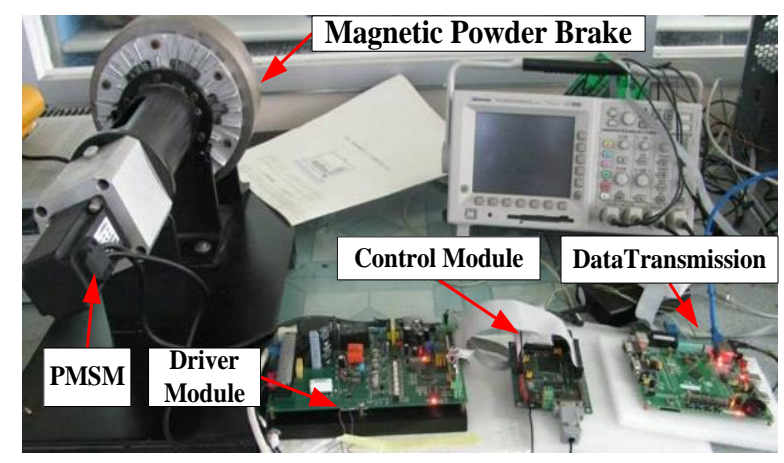

Figure 3. PMSM Experiment Systems

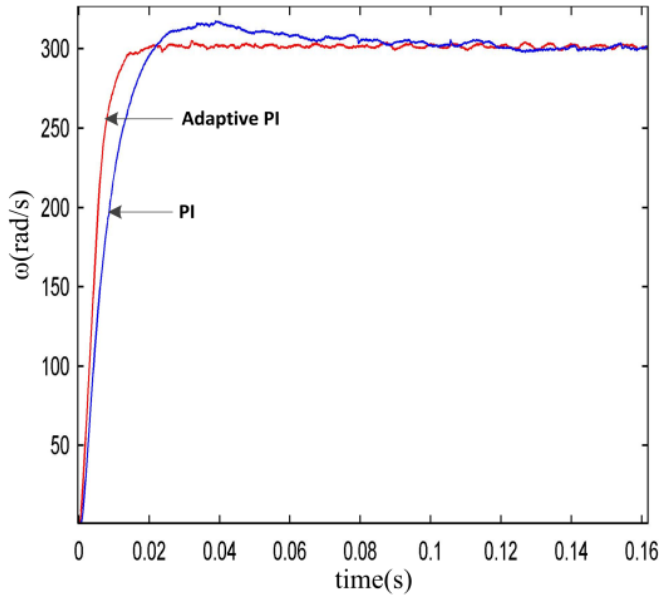

(a) No-load Torque

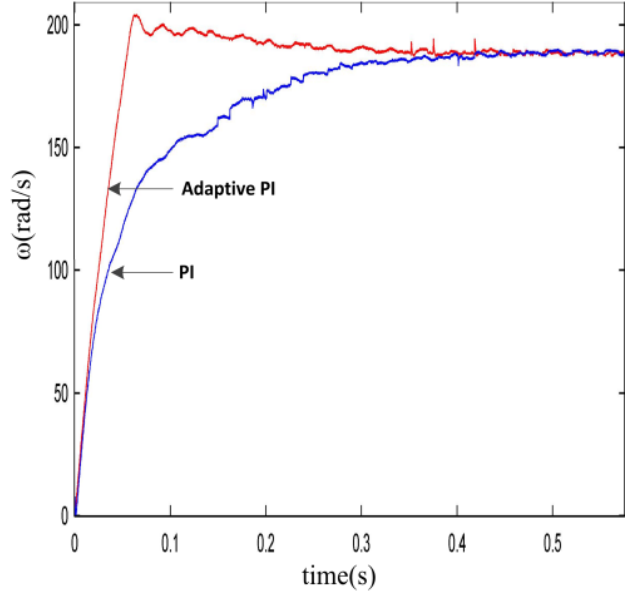

(b) Rated Load Torque

Figure 4. Curve of Tracking Speed with Two Control Methods

Under a no-load disturbance, a speed command is 300 rad/s. Figure 4(a) shows that the proposed controller can track more quickly the rated speed and the overshoot is smaller than PI controller. Due to the moment of inertia of PMSM servo experiment system being large as rated load torque, the response of PI controller becomes slow shown in Figure 4(b). The speed signal is got by $\mathrm{M} / \mathrm{T}$ speed detection method in the experiment. The reference slow speed is $10 \pi \mathrm{rad} / \mathrm{s}$ and the speed curve is original signal without a low-pass filter in Figure 5. Obviously, the steady state error of two control method is $3 \mathrm{rad} / \mathrm{s}$ for the proposed controller and 10 rad/s for PI controller. The slow speed performance for the 
proposed controller is better than PI controller.

Figure 6 shows the online estimated value of the parameters and the load torque. The error of equation (2) can be quick to approximately converge zero and it satisfies the sufficient condition of the proposed model adaptive compensation algorithm. The estimated value of the other parameters need not converge to the real value.

In Figure 7, external force $0.2 \mathrm{~N} . \mathrm{m}$ is applied to the experiment system. Due to the load torque disturbance, the speed output of PI control can not track a given reference speed and there is a big error in terms of the load torque disturbance shown as Figure 7(a). The proposed controller can overcome quickly the reference speed and the error is smaller shown as Figure 7(b).

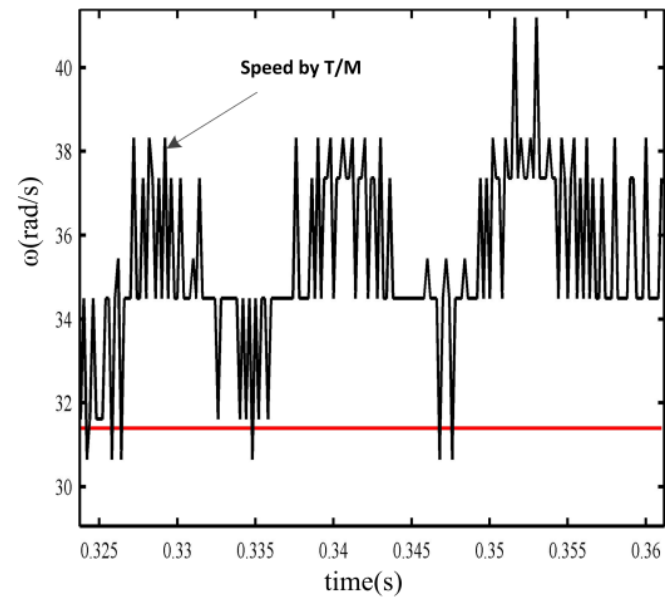

(a) No-load Torque

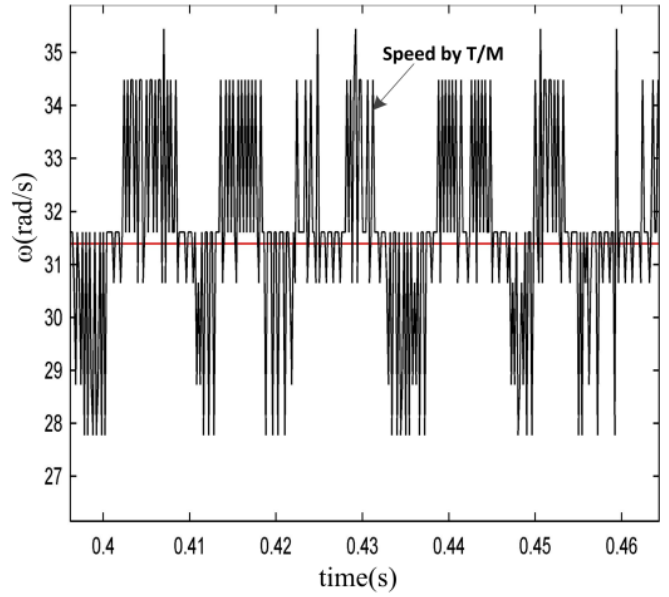

(b) Rated Load Torque

Figure 5. Curve of Tracking Speed with Two Control Methods
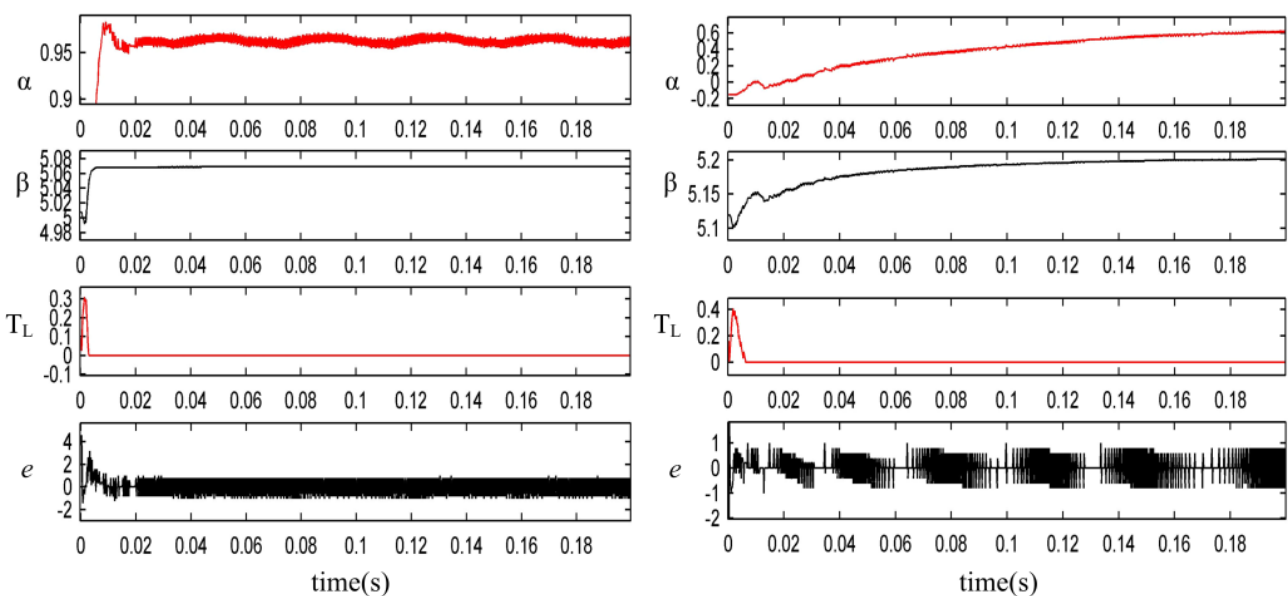

(a) No-load Torque

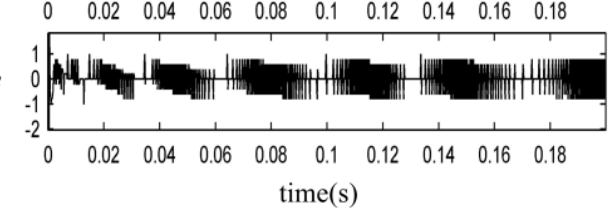

(b) Rated Load Torque

Figure 6. Curve of Online Estimated Parameters and Error 


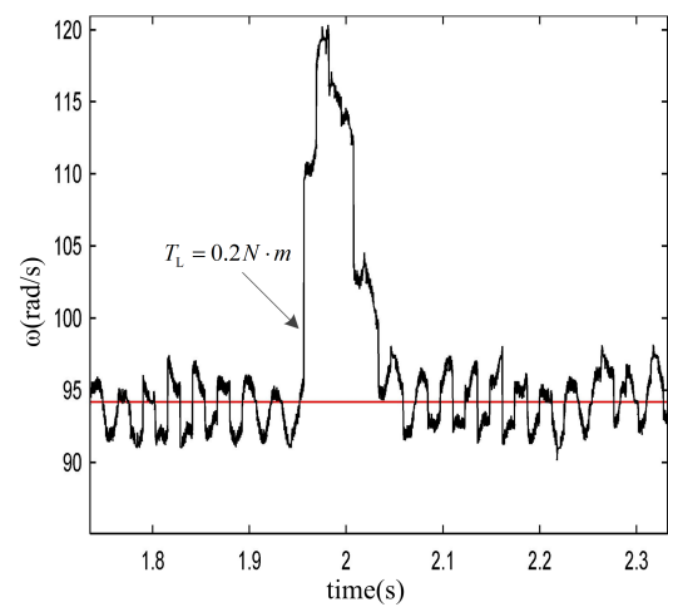

(a) PI

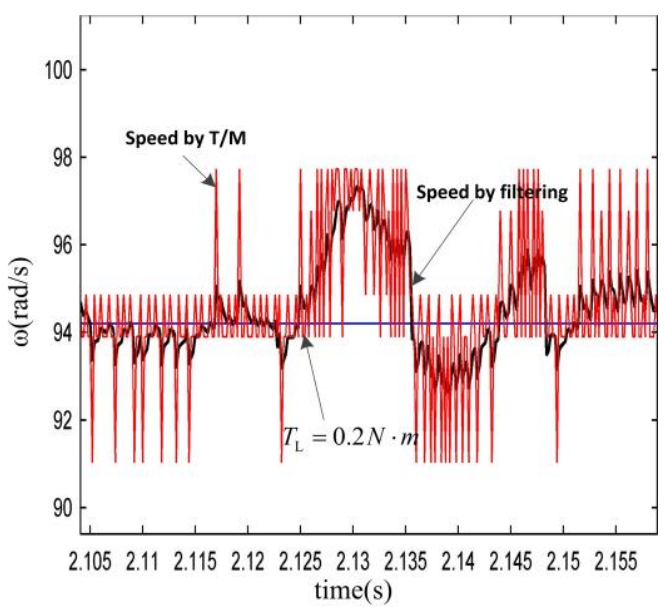

(b) Adaptive Control

Figure 7. Curve of Tracking Speed with Two Control Methods

\section{Conclusion}

Carrying out simulation and experimental studies under different conditions of PMSM speed control system, the proposed adaptive control can overcome well the load torque disturbance and parameter variations of PMSM speed control system. Especially the good performance of the slow speed control is also verified. So the result of experimental studies indicates the proposed control scheme has superior performance for the speed tracking. And constituting structure with online computing is extremely simple and easy to implement in industrial drives and machine arm drive.

\section{Acknowledgments}

The authors are very thankful to Fujian Province Educational Department for its support of this work (project No.JA14247), and also thankful to Fujian Province Technology Department for its support of this work (project No.2013H0037, No.2013J01204).

\section{References}

[1] R. D. Lorenz, T. A. Lipo and D. W. Novotny, "Motion Control with Induction Motor", Proc. Int. IEEE, vol. 82, (1994), pp. 1215-1240.

[2] K. Y. Wang, J. Chiasson, M. Bodson and L. M. Tolbert, "A Nonlinear Least-squares for Identification of the Induction Motor Parameter", IEEE Transactions on Automatic Approach Control, vol. 50, no. 10, (2005), pp. 1622-1628.

[3] C. Zhenfeng, Z. Yannu and L. Jie, "Online Adaptive Parameter Identification for Interior Permanent Magnet Synchronous Motor Drive", Electric Machines and Control, vol. 14, no. 4, (2010), pp. 9-13.

[4] Z. Zedong, L. Yongdong, X. Yi and M. Fadel, "Load Torque Observer of Permanent Magnet Synchronous Motor", Transactions of China Electro Technical Society, vol. 25, no. 2, (2010), pp. 29-36.

[5] Y. M. Menaa, O. Touhami and R. Ibtiouen, "Estimation of Rotor Resistance of an Induction Motor Using Extended Kalman Filter and Spiral Vector Theory", Proc. Int. IEEE Control Applications, vol. 2, (2003), pp. 1262-1266.

[6] G. Xiwen, W. Junjing, L. Guoli, H. Cungang and Q. Zhe, "Adaptive Fuzzy Control for Permanent Magnet Spherical Motor Based on Friction Compensation", Proceedings of the CSEE, vol. 31, no. 15, (2011), pp. 75-81.

[7] L. Ying, Z. Bo and F. Sichen, "Sliding Mode Control of PMSM Based on A Novel Disturbance Observer", Proceedings of the CSEE, vol. 30, no. 9, (2010), pp. 80-85.

[8] L. Zhigang, W. Junzhen and Z. Jiangbo, "Neural Adaptive Sliding Mode Control for Permanent Magnet Synchronous Motor", Electric Machines and Control, vol. 13, no. 2, (2009), pp. 290-295.

[9] C. Roug, D. Zhiquan and Y. Yaugguang, "Research on Resisit-disturbance Performance of Servo System Based on Load Torque Observer", Proceedings of the CSEE, vol. 24, no. 8, (2004), pp. 103-108. 
[10] Y. Zhang, C. Akujuobi, W. Ali, C. L. Tolliver and L. San Shieh, "Load Torque Disturbance Resistance Speed Controller Design for PMSM", IEEE Transactions on Industrial Electronics, vol. 53, no. 4, (2006), pp. 1198-1209.

[11] C. Yi Huan and T. Chi Chen, "Robust Control of Induction Motor with A Neural-network Load Torque Estimator and A Neural-network Identification”, IEEE Transactions on Industrial Electronics, vol. 46, no. 5, (1999), pp. 990-997.

[12] Y.A.-R. I. Mohamed, "Adaptive Self-tuning Speed Control for Permanent Magnet Synchronous Motor Drive with Dead Time”, IEEE Transactions on Energy Conversion, vol. 21, no. 4, (2006), pp. 855-862.

[13] P. S. R. Diniz, “Adaptive Filtering: Algorithms and Practical Implementation”, Springer, New York, (2002).

\section{Authors}

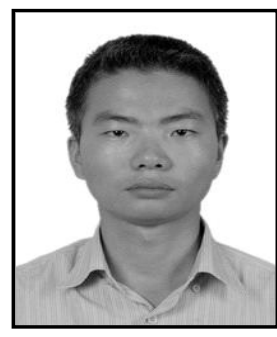

Jiang Xuecheng, currently working as Associate Professor in Minjiang University, China. He received his $\mathrm{PhD}$ from Xiamen University in 2012. Current areas of interests are adaptive control theory, system parameter identification, motion control and electrical engineering and automation. E-Mail: jxc603@aliyun.com.

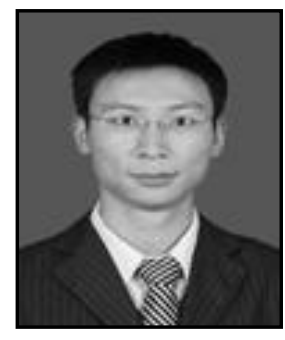

He Dongwei, currently working as Lecture in Fujian University of Technology, China. Current areas of interests are intelligent control, robots control and optimization and information optical technology. He obtained his PhD from Xiamen University in 2014. 